International Journal of Advanced Trends in Computer Science and Engineering

Available Online at http://www.warse.org/IJATCSE/static/pdf/file/ijatcse13762018.pdf

https://doi.org/10.30534/ijatcse/2018/13762018

\title{
Performance analysis of WRP with mobile sink based on data collection to base station in a WSN
}

\author{
${ }^{1}$ R.Tamil Kodi, ${ }^{2}$ L.Venkateshwara Kiran, ${ }^{3}$ V.L.Sailaja, ${ }^{4}$ M.V.Subba Rao \\ ${ }^{1,2}$ Department of MCA, ${ }^{3,4}$ Department of HBS \\ Godavari Institute of Engineering \& Technology, Rajahmundry, A.P,India.
}

\begin{abstract}
This research paper proposes the wireless sensor network (WSN) is collect information for sensor nodes. A sensor hub blankets every one data which will be display in its sensing go. With get the data exhibit on some other sensor range, the networks use a transform known as directing. A standout amongst those directing issues in remote sensor system (WSN) will be concerned for expanding those sensor system lifetime same time ceaselessly directing the gathered information (information) of the build station (central server). A course (sometimes called concerning illustration Path) may be a situated of sensors that build a association the middle of An sourball hub and a sink hub (base station). The directing may be used to figure out a situated about different routes with most extreme total apples and oranges lifetime same time compelling the existence of every sensor by its starting battery life. Previously, WSN, we request a Vitality productive way which may be used to send gathered majority of the data of the focus base station. At that base station, the accepted information would transform further. Late developments for remote sensor networks need prompted a number new conventions particularly outlined to sensor networks the place vitality mindfulness may be a crucial thought. A vitality productive technique to vitality adjusted directing to WSN is recommended in this paper. In this method, the next-hop hub may be chose as stated by the attention to connection weight and ahead vitality thickness. The effectiveness from claiming suggested vitality mindful directing strategy is demonstrated through MATLAB reproduction outcomes.
\end{abstract}

Keywords: Energy balanced routing, wireless sensor network

\section{INTRODUCTION}

Wireless sensor networks (WSNs) would create of a vast number for sensor hubs deployed done a field as shown in figure 1 . They need wide-ranging applications, some about which incorporate military [1]-[3], nature's domain observing [4], [5], farming worker [6], [7], home mechanization [8], keen transportation [9]-[8], and wellbeing . Each sensor hub need those proficiency should gather What's more methodology data, and to forward whatever sensed information back with one or that's only the tip of the iceberg sink hubs through their remote transceiver to a multi jump way. Clinched Alongside addition, it will be provided for a battery, which might make troublesome or illogical will replace, provided for those amount for sensor hubs and deployed earth. The greater part of the genuine networks from claiming IA, free from claiming their age, function, and scope, meet on comparative architectures. Along these lines scientists attempted with manufacture a bound together model to intricate networks in the last decades.

That way of a versatile sink relies on the constant prerequisite about information prepared toward hubs. To example, previously, hard ongoing provisions for example, a fire-detection system natural information necessity will make gathered Eventually Tom's perusing a versatile sink rapidly. Moreover, a mobile-sink hub might transform its position after a specific time of time and select in turn information collection/ practical site. Those practical locales What's more comparing stay time need aid subject to the lingering vitality for sensor hubs [8]

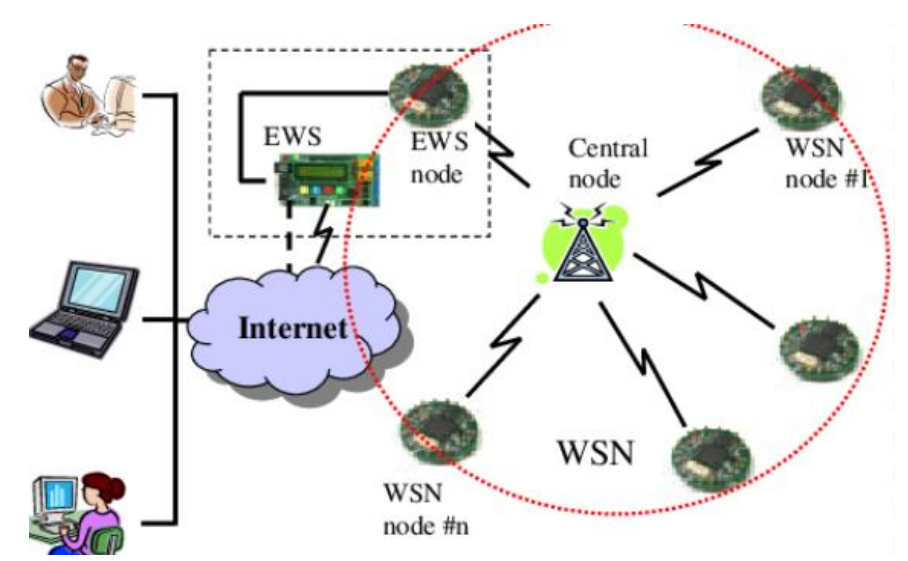

Figure 1: The components of a sensor node 


\section{RELATED WORKS}

\subsection{Sink Mobility Scheme and Optimized Networks Lifetime}

Here they used two-level fuzzy logic for sink mobility scheme to optimize networks lifetime and energy consumption. Qualified hubs to directing need aid chosen dependent upon their vitality and number from claiming neighbors. Each SINKS need aid actualized for amount for freely altered alternately versatile wanted area inside the organize field. Upgrading Vitality utilization and expanding the organize lifetime would those primary concern in remote sensor networks the place hubs vitality need aid basically utilized to sending information of the sink alternately build station. Number directing conventions are introduced with spare vitality [4-5] to information gathering Furthermore utilizing settled base station issues need aid sincerely distinguished. In this paper, we point to display an sink portability plan with streamline networks lifetime and Vitality utilization utilizing An two-level fluffy rationale. Specifically, those versatile sink moves towards a fruitful top banana group and diminishes the Main group Vitality utilization. In the main fluffy level, qualified hubs would chosen dependent upon their vitality What's more amount for neighbors? At that point in the second fluffy level, sets about effective hubs would comprehensively assessed in the organize for two period parameters. We bring also tried those impact about system size, sink development to a irregular way alternately done An regulated development around square course organize. The Outcomes indicate that square routes beat the others and save greater amount vitality in the whole organize. Sink route, numerous foreordained routes [7] bring been recognized here. At whatever point connection disappointment occurs, that upstream hub instantly identifies the animated neighbor of the connection. This will be rehashed until the hotspot hub achieves the join. At that point course recuperation procedure will be initiated.

\subsection{Low-Energy Adaptive Clustering Hierarchy}

LEACH may be those initial What's more the majority prevalent Vitality productive hierarchic grouping calculation to WSNs that might have been recommended to decreasing control utilization. On LEACH, those grouping errand will be turned around those nodes, In light of span. Regulate correspondence is utilized Eventually Tom's perusing each group head $(\mathrm{CH})$ should forward the information of the build station (BS). It utilization groups on prolong those term of the remote sensor organize. Drain may be In light of a amassed (or fusion) system that combines alternately aggregates the first information under An more diminutive measure of information that convey just serious data should the greater part distinctive sensors. Drain isolates the An organize under a few group for sensors, which need aid constructed by utilizing restricted coordination Furthermore control not best to decrease the measure of information that need aid transmitted of the sink, as well as to aggravate directing Furthermore information spread a greater amount versatile also strong.

\section{PROPOSED MODEL}

\subsection{Energy Aware Routing Protocol}

A routing protocol tags how routers convey for each other, scattering majority of the data that empowers them will select routes the middle of at whatever two hubs for a workstation organize. Directing calculations determine that particular decision from claiming course. Every switch need a from the earlier information just for networks joined to it straightforwardly. A directing protocol offers this majority of the data main "around prompt neighbors, et cetera All around those system. This way, routers pick up information of the topology of the organize.

\subsection{Network Model}

The WSN comprises about $\mathrm{n}$ hubs which would static Furthermore uniformly dispersed once a square field utilizing a homogeneous Poisson dissemination with hub thickness $\rho$. That correspondence may be symmetric, and two hubs inside the transmission span $\mathrm{r} 0$ need aid neighbors in the system. To guarantee the organize connectivity, we Accept that the amount for neighbors for node, characterized Similarly as hub degree d(i), may be fulfilled by those states On [1]. Nc hubs in the. Event-sub-network would gather under groups. So as with assess execution under Perfect directing condition, we expect immaculate Macintosh states Furthermore connection caliber. Besides we utilize the radio model as takes after:.

Etx $=\mathrm{k}$ Eelc $+\mathrm{k}$ Eampd $^{2}$ and Erx $=\mathrm{k}$ Eelc

The WSN comprises about $\mathrm{n}$ hubs which would static Furthermore uniformly dispersed once a square field utilizing An homogeneous Poisson dissemination with hub thickness $\rho$. That correspondence may be symmetric, and two hubs inside the transmission span r0 need aid neighbors in the system. To guarantee the organizee connectivity, we Accept that the amount for neighbors for node, characterized Similarly as hub degree d(i), may be fulfilled by those states On [1]. Nc hubs in the. Event-sub-network would gather under groups. So as with assess execution under Perfect directing condition, we expect immaculate $t$ states Furthermore connection caliber. Besides we utilize the radio model as takes after:

The first vitality of the hub can be a chance to be acknowledged Likewise E0. The point when this vitality is emptied implies hub dies, however vitality of the sink hub could make included. The area of the sink Furthermore sensor hub could make altered.

Those sink hub show the message on at sensor hubs in the sensing field. Gained sign quality is used to figure that separation between hotspot and sink hub. Vital hub can't make assigned at first. It can be chose then afterward the topology improvement [8]. The communication range of the sensor node is set to $\mathrm{d} 0$. The threshold $\mathrm{d} 0$ can be defined as $\mathrm{d} 0=\sqrt{ }(\varepsilon f \mathrm{~s} 2 / \varepsilon \mathrm{mp})$ where $\varepsilon f \mathrm{fs}, \varepsilon \mathrm{mp}$ are energycoefficients

$\mathrm{d}(\mathrm{i}$, sink $)$ is the distance between the node $\mathrm{i}$ and sink node. $\mathrm{d}(\mathrm{i}$, sink) can be defined as

$$
\mathrm{R}=(\mathrm{X}, \sqrt{ }((\mathrm{H} / 2) 2+(\mathrm{X}+\mathrm{W}) 2))
$$


When $i$ is the cluster head, the communication radius of the cluster head Ropt(i) is given by

$$
\operatorname{Ropt}(\mathrm{i}) \sim \mathrm{f1}(\mathrm{R}) \text {. }
$$

Where $\mathrm{f} 1(\mathrm{~d}(\mathrm{i}$, sink $)$ is the function of $\mathrm{d}(\mathrm{i}$, sink $)$. And its ranges are between 0 and $\mathrm{d} 0$.

\subsection{Mobile Sink}

It's an vitality productive method for information gathering done remote sensor networks. In this paper, we recommend a mixture unconstrained development example to a portable sink for the point about adjusting the Vitality utilization about sensor hubs. Our approach makes the Emulating contributions, as compared with those fill in news person in the writing.. Here maximum energy dissipation happened only at single node in the network and the average energy dissipation over all nodes will get reduced considerably.

\subsection{Energy Aware Routing Protocol}

A join will be used to associate two hubs. Whether that separation the middle of two hubs is less that transmission range then we could make a connection the middle of them. Each connection in our model will be a bidirectional link, this may be actualized with two one-directional join. Every hub need in any event person clinched alongside connection also particular case crazy join. Each joins need its own properties for example, expense. TX Node and RX Node shown in figure 2. Note that Cost properties are explained in routing section. For each link TX Node and RX Node show which node is transmitter and which node is receiver of that specific link.

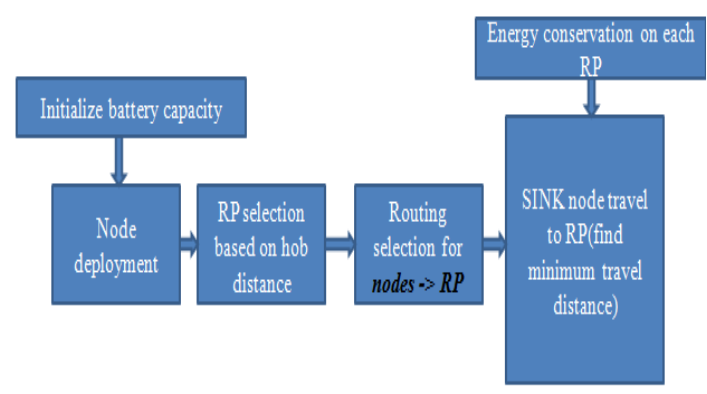

Figure.2. Proposed energy aware sink routing architecture

\subsection{Link Cost}

Those elementary relic from claiming vitality mindful WSN may be known as join expense (or hub cost) Cuv of a connection $(\mathrm{u}, \mathrm{v})$. With figure out Cuv, those taking after four parameters, viz. , transmission expense from laiming hub u (Etx), gathering expense of hub $\mathrm{v}$ (Erx) and the lingering vitality for hubs what's to come for $\mathrm{U}$ and $\mathrm{v}$ (REu Also rev respectively), might make obliged. It might be said here that the initially two parameters need aid all things considered identifier as vitality proficiency.

\section{RESULTS \& ANALYSIS}

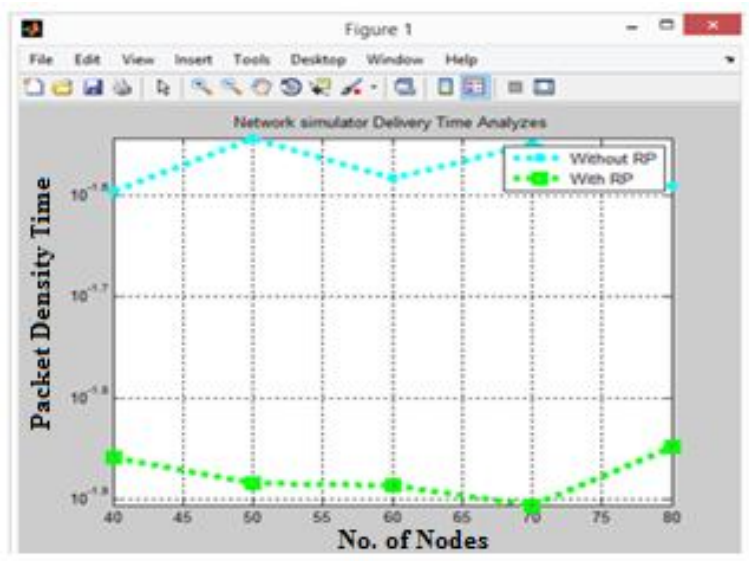

Figure 3: Trade off analyzes of nodes vs. delivery ratio

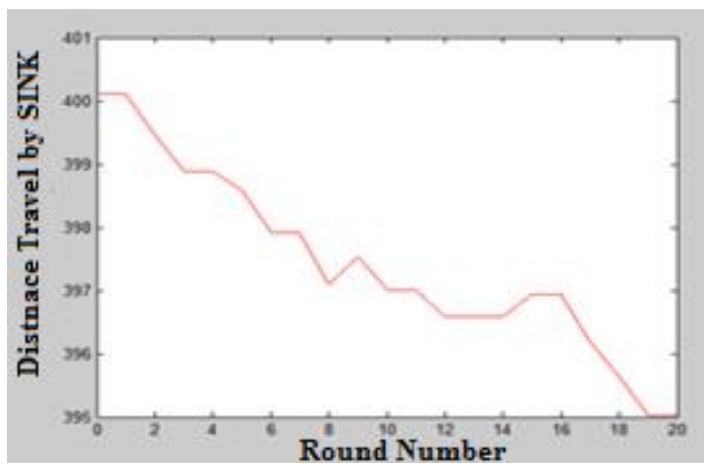

Figure 4: SINK code travelling distance

As in figures $3,4 \& 5$ after setting expense should every link, we could figure the base way expense starting with each hub with sink Eventually Tom's perusing utilizing a recursive way expense capacity. In this capacity expected that way cosset about sink will be zer0

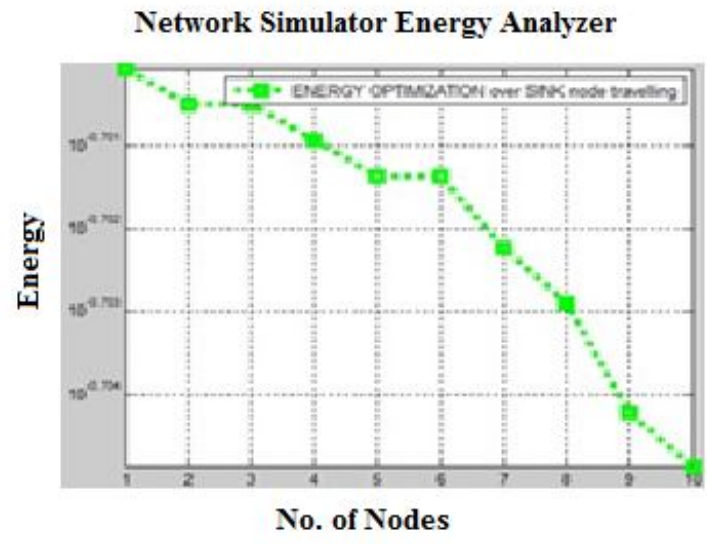

Figure 5: Network energy analyzes 


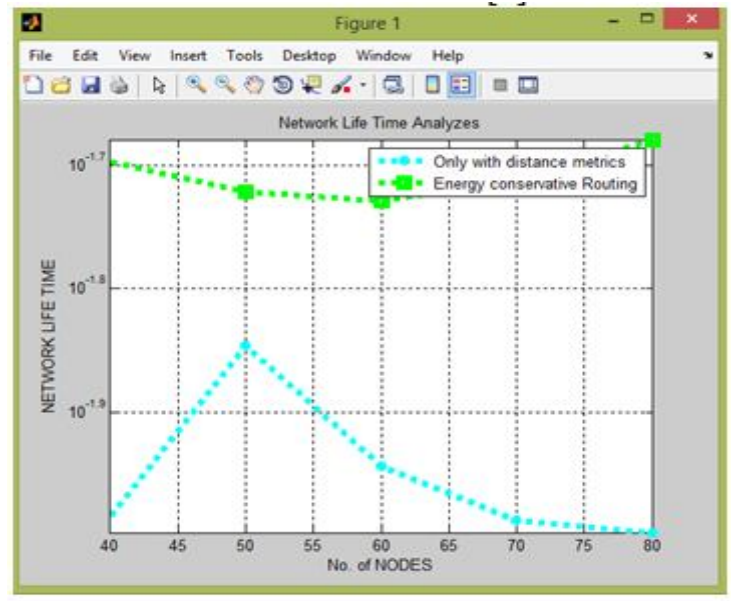

Figure 6: Network life time analyzes

At whatever point a hub accept signal from other hub recomputed those base way cosset of the sink. For registering cosset capacity clinched alongside algorithms as in figures 5 and 6 , tought bring exactly information regarding bundle energy, accessible vitality.

\section{CONCLUSION}

In this paper, we analyzed the performance of WRP with mobile sink based data collection to base station in a WSN. The efficiency of weight age based selection of RPs for the low energy expenditure of sensor nodes and to ensuring sensed data is collected on time. In addition, visiting virtual nodes which are considered to be a RP point with minimum traveling distances through extensive search for minimum path distance along with energy conservation as benchmark for each iteration. Our simulation results proved the efficiency of weight age based RP selection for minimized delay and energy optimized sink node visits to RP points. To extend the analyzes of trade-off between number nodes used over network life time over throughput rate with balanced energy aware routing.

\section{REFERENCES}

[1] I. F. Akyildiz, W. Su, Y. Sankarasubramaniam, and E. Cayirci, "Wireless sensor networks: A survey," Comput.Netw., vol. 38, no. 4, pp. 393-422, Mar. 2002. https://doi.org/10.1016/S1389-1286(01)00302-4

[2]. S. Diamond and M. Ceruti, "Application of wireless sensor network to military information integration," in Proc. 5th IEEE Int. Conf. Ind. Inform., Vienna, Austria, Jun. 2007, vol. 1, pp. 317-322. https://doi.org/10.1109/INDIN.2007.4384776

[3]. I. Bekmezci and F. Alagz, "Energy efficient, delay sensitive, fault tolerant wireless sensor network for military monitoring," Int. J. Distrib. Sens. Netw., vol. 5, no. 6, pp. 729-747, 2009. https://doi.org/10.1080/15501320902768625

[4]. A. Mainwaring, D. Culler, J. Polastre, R. Szewczyk, and J. Anderson, "Wireless sensor networks for habitat monitoring," in Proc. 1st ACM Int. Workshop Wireless Sens. Netw. Appl., New York, NY, USA, Sep. 2002, pp. 88- 97. https://doi.org/10.1145/570738.570751

[5]. J. Zhang, W. Li, Z. Yin, S. Liu, and X. Guo, "Forest fire detection system based on wireless sensor network," in Proc. 4th IEEE Conf. Ind. Electron. Appl., Xi'an, China, May 2009, pp. 520-523. https://doi.org/10.1109/ICIEA.2009.5138260

[6]. L. Ruiz-Garcia, L. Lunadei, P. Barreiro, and I. Robla, "A review of wireless ssensor technologies and applications in agriculture and food industry: State of the art and current trends," Sensors, vol. 9, no. 6, pp. 4728-4750, Jun. 2009. https://doi.org/10.3390/s90604728

[7]. N. Wang, N. Zhang, and M. Wang, "Wireless sensors in agriculture and food industry-recent development and future perspective," Comput. Electron. Agriculture, vol. 50, no. 1, pp. 1-14, Jan. 2006. https://doi.org/10.1016/j.compag.2005.09.003

[8] Anilgantala, Dasari Swathi, J Sravana, Paparao Nalajala," Automatic License Plate Detection And Character Recognition In License Plate International Journal Of Advanced Trends In Computer Science And Engineering, Vol.5 , No.1, Pages : 46-50, (2016)

[9] Goodubaigari Amrulla, Murlidher Mourya, Rajasekhar Reddy Sanikommu,"A Survey of:Securing Cloud Data under Key Exposure", International Journal of Advanced Trends in Computer Science and Engineering, Volume 7, No.3, Pp-30-33, 2018. 